\title{
Native and Degraded Forms of Sweet Potato Starch Phosphorylase ${ }^{\dagger}$
}

\author{
Chui-Liang Chiang, Yu-Ling Lu, Rong-Huay Juang, \\ Ping-Du LeE and Jong-Ching SU \\ Department of Agricultural Chemistry, National Taiwan University, \\ I Roosevelt Road Section 4, Taipei, Taiwan 10764, ROC \\ Received April 27, 1990
}

\begin{abstract}
By immunoblotting using monoclonal and polyclonal mouse antibodies against sweet potato (Ipomoea batatas (L.) Lam.) starch phosphorylase (SP), and by activity and dye staining methods, fresh extracts obtained from sweet potatoes immediately after harvest or from those being stored under various conditions were analyzed for SP by PAGE. Freshly prepared pure or crude SP, or those stored under various conditions were also analyzed. We found that the native enzyme from either of the cultivars Tainong 57 or 65 was $220-\mathrm{kDa}$ in size and constituted of two 112-kDa subunits. Either in vivo or in vitro, storage caused degration of the enzyme to two groups of small subunits of about 50-kDa each. On prolonged cold storage of purified enzyme in $50 \%$ glycerol, the formation of a small amount of a $250-\mathrm{kDa}$ protein was detected. It had a $150-\mathrm{kDa}$ subunit that was formed presumably by transpeptidation catalyzed by a contaminating protease. This $250-\mathrm{kDa}$ form had the primed SP activity only. The SP from the two kinds of cultivar showed considerable differences in the proteolytic susceptibility and peptide map. A part of Tainong 65 SP was supposed to be a heterodimer.
\end{abstract}

Plant starch phosphorylase (SP) $(\alpha-1,4$-glucan:orthophosphate $\alpha$-D-glucosyltransferase, EC 2.4.1.1) is generally regarded as a starch degrading enzyme, and has been obtained from spinach, banana, potato, sweet potato, algae, etc. ${ }^{1)}$ It has also been reported, however, that the enzyme may act in starch synthesis. ${ }^{2)}$ We have reported that the enzyme as well as its noncompetitive inhibitor $\beta$-amylase (BA) were localized in the starch synthesizing amyloplasts of sweet potato roots ${ }^{3,4)}$ and the enzyme protein content was proportional to the starch content that ranged from 7 to $23 \%$ in about 60 varieties. ${ }^{5)}$ These findings indicated that the role played by SP in the starch-accumulating tissue needs more investigation. The plant SP has been known to occur in multiple forms, and its population composition changes depending on the physiological state of the source material. ${ }^{61}$ Proteolysis was suggested as a cause of multiforms of the potato enzyme. ${ }^{7)}$ We studied the subunit patterns of SP prepared from fresh and stored roots of the sweet potato cultivar Tainong 65 (T65, formerly cited as Tainon 65 in ref. 3, by Western analysis using monoclonal antibodies as probes and concluded that the sweet potato SP was comprised of two identical subunits that were susceptible to the attack of proteases at the central part of the peptide chain. ${ }^{3)}$ Our data also showed the presence of an intact large subunit even when the SP was degraded to about $80 \%$ of its original size when the enzyme source material was stored at room temperature for over three months. This strongly indicated the presence of a protease-resistant subunit structurally different from the protease-sensitive one. However, as the work was extended by

+ This work was supported by grants from the National Science Council, Republic of China.

Abbreviations: SP, starch phosphorylase; BA, $\beta$-amylase; T57 and T65, cultivars Tainong 57 and Tainong 65 of the sweet potato Ipomoea batatas (L.) Lam., respectively; PMSF, phenylmethylsulfonyl fluoride; ELISA, enzyme-linked immunosorbent assay. 
using different cultivars, we found that the enzyme prepared from the cultivar Tainong 57 (T57) often had no "intact" large subunit, causing suspicion that it may have some "intact" small subunits as the primary gene products. Information concerning the native size of the enzyme molecule is so important in guiding our cDNA cloning work that we tried to clarify the situation by making a comparative PAGE study of SP with respect to the differences in: 1) source cultivars; 2) ages of sources; 3) modes and durations of source storage; and 4) storage durations of purified enzymes.

\section{Materials and Methods}

Sweet potato samples. Either freshly harvested or stored roots of sweet potato [Ipomoea batatas (L.) Lam.], cv. Tainong 57 (T57) and cv. Tainong 65 (T65), were used.

Enzyme purification. All purification steps were done by the procedure described previously up to the gel filtration step $^{3)}$ except the $50 \mathrm{~mm}$ imidazole- $\mathrm{HCl}$ buffer for enzyme extraction contained $1 \%$ insoluble PVP, $0.5 \%$ ascorbic acid, $1 \mathrm{~mm} \beta$-mercaptoethanol, and a mixture of protease inhibitors (PMSF $0.1 \mathrm{~mm}$, leupeptin $1 \mu \mathrm{M}$, EDTA-2Na $0.1 \mathrm{~mm}$, aprotinin $0.2 \mu \mathrm{M}$, pepstatin $0.1 \mathrm{~mm}$, and $\mathrm{PCMB}$ $1 \mu \mathrm{M})$, if necessary, in addition to $1 \mathrm{~mm}$ dithiothreitol, $0.1 \mathrm{~mm}$ EDTA, and $10 \% \mathrm{w} / \mathrm{v}$ sucrose. The active fractions from the gel filtration column were pooled and concentrated in an Amicon unit. The concentrate was further purified, if necessary, by running preparative discPAGE in a $7.5 \%$ gel with a BRL V-16 apparatus at $4^{\circ} \mathrm{C}$ and $120 \mathrm{~V}$ for $10 \mathrm{hr},{ }^{8)}$ The active band found by guiding strip staining and seen under a UV lamp was sliced out and immersed in Tris-acetate buffer, $\mathrm{pH} 8.6$. The protein in the gel slice was recovered in an ISCO Model 1750 electroeluation apparatus.

Enzyme assay. SP activity was assayed in the direction of starch synthesis. ${ }^{3)}$ Protein was measured by the Folin-phenol reagent using bovine serum albumin as the standard. ${ }^{\text {?) }}$

Our previous experiences had shown that the in vivo degradation of SP to the state of having 50-kDa peptides as the major subunits had little or no effect on its catalytic activity. ${ }^{3)}$ In addition, since BA coexisting in a large amount in the enzyme extract is an inhibitor of $\mathrm{SP},{ }^{4)} \mathrm{SP}$ activity assays were done only for the purpose of enzyme purification.

Preparation of and use in ELISA of SP-specific monoclonal and polyclonal antibodies. Mouse ascites fluids containing monoclonal and polyclonal antibodies were prepared and used in ELISA by the procedures reported previously. ${ }^{10 \%}$ Two clones of monoclonal antibodies, G2c, an $\mathrm{IgG}$, and $\mathrm{J} 3 \mathrm{~b}$, an $\mathrm{IgM}$, were selected from our stock for use.

Peptide mapping. A solution of the enzyme in the native state was digested by one-tenth volume of one of protease solutions at $37^{\circ} \mathrm{C}$ for $1 \mathrm{hr}$. The protease solutions used were $1 \%$ trypsin, $1 \%$ chymotrypsin, $0.5 \%$ protease $\mathrm{V}_{8}$, and $0.5 \%$ subtilisin. The reaction was stopped by adding an equal volume of SDS and $\beta$-mercaptoethanolcontaining buffer and putting the mixture into a boiling water bath for $5 \mathrm{~min}$. The digest was run in $15-20 \%$ gradient SDS-PAGE ${ }^{11}$ and the peptide bands in the gel were colored by a silver stain. ${ }^{12)}$ For the Western analysis of either native proteins or peptide fragments, immunoblotting and immunostaining methods previously reported were followed. ${ }^{3)}$

Molecular weight measurement. The molecular sizes of native enzymes were compared by gel filtration and 5-12\% gradient (pore-limit) PAGE ${ }^{13)}$ while the molecular masses of enzyme subunits and peptide fragments were measured by $7.5-15 \%$ gradient SDS-PAGE. ${ }^{11}$

\section{Results and Discussion}

\section{Enzyme purification}

The bulk of SP was salted out in $2545 \%$ ammonium sulfate. Through the steps of ion-exchange (DEAE-Sepharose) and gel filtration chromatographies (Fig. 1A), electrophoretically homogeneous SP could be obtained.

\section{Characterization by antibodies}

When the eluate from a gel filtration column was monitored simultaneously with ordinary UV and activity assays (Fig. 1A) and ELISA using a polyclonal and two monoclonal antibodies (Fig. 1B), it was found that a greatly degraded yet slightly active form of the enzyme was present and it could be strongly recognized by the monoclonal antibody G2c but not by J3b. As we will see later, the sweet potato SP may be degraded by protease in vivo. Since the main activity peak could be recognized distinctly by all antibody preparations, we may say that the epitope recognized by $\mathrm{J} 3 \mathrm{~b}$ is lost in the proteolysis. Figure $1 \mathrm{~B}$ also indicated that the $\mathrm{G} 2 \mathrm{c}$ clone had so strong a binding affinity toward the SP that even a very small amount 
of aggregated form of the enzyme (peak at the void volume) could be detected by it.

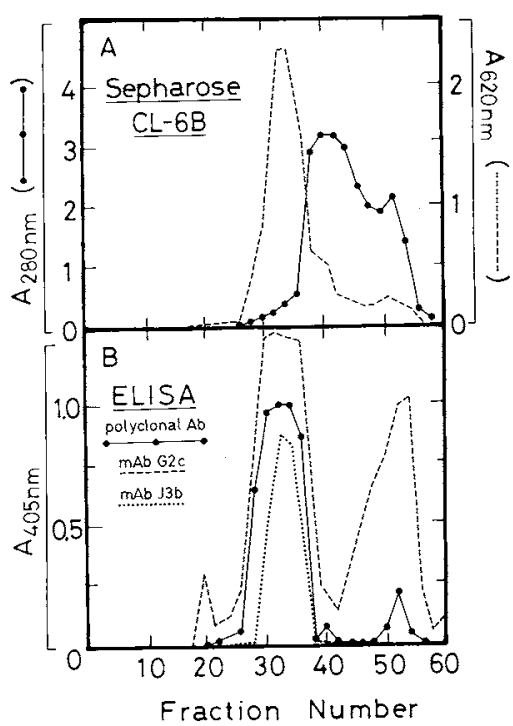

Fig. 1. Chromatographic Patterns of Phosphorylase from T65 Sweet Potatoes

A and B. Gel filtration on Sepharose CL-6B: column size, $2.6 \times 25 \mathrm{~cm}$; elution rate, $24 \mathrm{ml} / \mathrm{hr}, 8 \mathrm{ml} / \mathrm{fraction}$.

Method of monitoring column eluates: A, activity assay and UV absorption; $B$, immunoassay with poly- and monoclonal antibodies.

\section{Forms of $S P$ in roots of different ages}

The fresh crude extracts, with or without the mixture of protease inhibitors added, of sweet potato $\mathrm{T} 57$ roots in sizes ranging from 1.5 to $6.5 \mathrm{~cm}$ in diameter, all showed a single band in disc-PAGE-Western blot patterns (data not shown), indicating that SP could maintain its intact form through developmental stages, and had the large subunit only.

\section{Changes of SP in vivo}

Crude extracts prepared from $\mathrm{T} 57$ roots stored at room temperature, $4^{\circ} \mathrm{C},-20^{\circ} \mathrm{C}$, or $-70^{\circ} \mathrm{C}$ for one, three, or five weeks were analyzed for SP by the SDS-PAGE-Western blotting method (Fig. 2). In addition, frozen solutions of a purified enzyme and a crude extract at $4^{\circ} \mathrm{C}$ were also stored and analyzed. In general, addition of a mixture of protease inhibitors to the extraction medium tended to enhance the image of the immunoblotted large subunit, suggesting the proteolytic nature of small subunit formation. It was interesting to find that SP freshly extracted from roots stored at room temperature or $4^{\circ} \mathrm{C}$ even for extended periods had a negligible amount of, while those obtained from the one stored under freezing

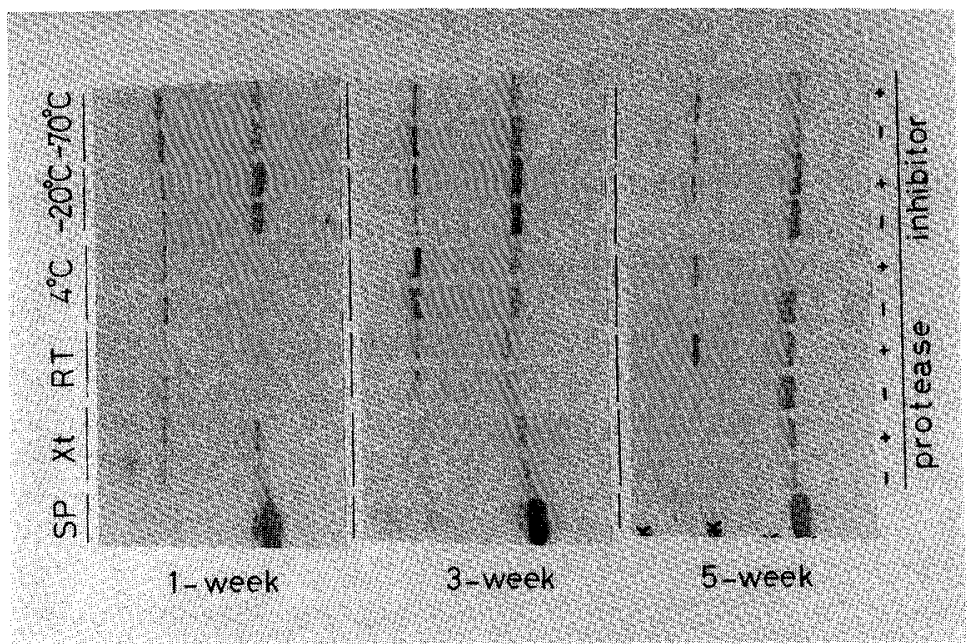

Fig. 2. 7.5-15\% SDS-PAGE/Western Blot Patterns of SP in Stored Extracts from Freshly Harvested T57 Roots, or in Fresh Extracts from Stored T57 Roots.

Monoclonal antibody $\mathrm{J} 3 \mathrm{~b}$ was used. SP, a purified preparation stored under freezing for about 6 months; $\mathrm{Xt}$, a crude extract stored at $4^{\circ} \mathrm{C} ; \mathrm{RT}, 4^{\circ} \mathrm{C}$, etc., fresh extracts from roots stored at room temperature, $4^{\circ} \mathrm{C}$, etc. The storage periods were 1,3 , and 5 weeks as indicated. Two parallel samples, with $(+)$ and without $(-)$ addition of protease inhibitors in the buffer, were run in each treatment. 
condition, $-20^{\circ} \mathrm{C}$, had a considerable amount of small subunits, the sign of degradation, after a storage period of as short as one week (see the upper right hand side of Fig. 2). These results may indicate that the proteolytic enzyme that degrades SP is physically separated from $\mathrm{SP}$ in vivo, and slow freezing of tissues in a laboratory freezer allows the formation in the cell of ice crystals big enough to destroy the subcellular compartment that separates SP from the protease. The degradation products of SP had about one-half the size of the parent molecule, confirming the previous finding that the central segment of the SP molecule was most susceptible to proteolysis.

\section{Changes of $S P$ in vitro}

When the crude enzyme extract was stored at either $4^{\circ}$ or $-20^{\circ} \mathrm{C}$ and analyzed at 3-day intervals, more extensive degradation of SP was observed at $4^{\circ}$ than at $-20^{\circ} \mathrm{C}$ while the BA band remained intact, indicating that SP was more susceptible than BA to the protease in the extract (data not shown). When purified $\mathrm{SP}$ in the imidazole buffer was stored at room temperature, $4^{\circ},-20^{\circ}$, or $-70^{\circ} \mathrm{C}$ in the presence or absence of the mixture of protease inhibitors for one to six weeks and analyzed by SDS-PAGE, a group of subunits of about $50-\mathrm{kDa}$ appeared and finally replaced the $112-\mathrm{kDa}$ one totally irrespective of whether protease inhibitors were present or not, although the inhibitors seemed to slow down the degradation (data not shown). These patterns of degradation were the same as those that took place in vivo. So we may say that the contaminating protease in the extract or the purified preparation could be the one that was responsible for in vivo degradation.

\section{Molecular masses of native T57 and T65 enzymes}

The relative molecular mass $\left(M_{r}\right)$ of the native forms of SP freshly prepared from T57 and $\mathrm{T} 65$ were estimated by gel filtration (Fig. 3A) and PAGE (Fig. 3B); the results obtained were 280 - and $330-\mathrm{kDa}$ respectively by the former and an identical value of $220-\mathrm{kDa}$ by the latter. $\mathrm{We}^{3)}$ and others ${ }^{14,15)}$ obtained anomalous molecular mass data for SP and other glycoproteins by gel filtration. We have shown that SP from T57 is a glycoprotein by the following: a) the purified enzyme, after being further separated by PAGE, gave a
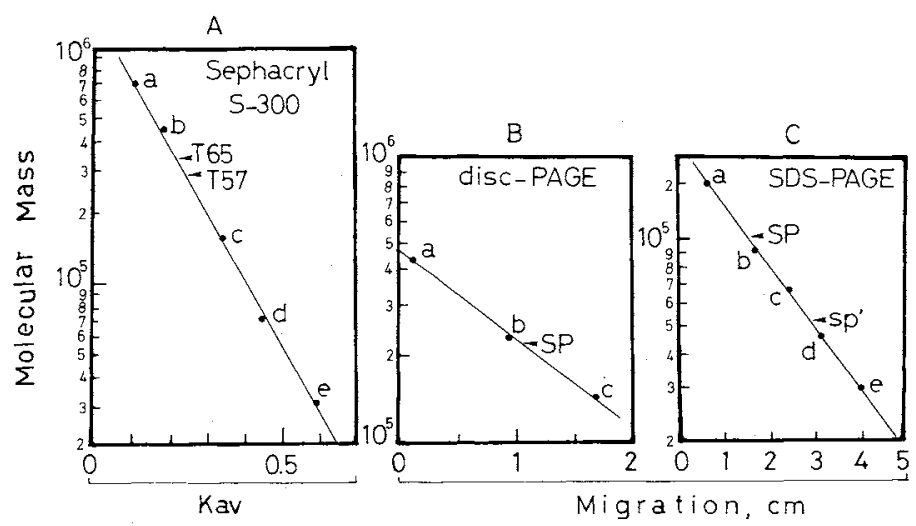

Fig. 3. Molecular Mass Determination of SP.

A. By gel filtration. Size of Sephacryl S-300 column, $1.6 \times 92 \mathrm{~cm}$. Standard proteins used (kDa): a, thyroglobulin, 669 ; b, ferritin, 440; $\mathrm{c}$, alcohol dehydrogenase, 150; $\mathrm{d}$, bovine serum albumin, 69 ; e, carbonic anhydrase, 29

B. By 5-12\% gradient (pore-limit) PAGE. Standard proteins used (kDa): a, ferritin, 440; b, catalase, 232; c, lactate dehydrogenase, 140.

C. By 7.5-15\% SDS-PAGE for subunits. Standard proteins used (kDa): a, myosin, 200; b, phosphorylase a, 92.5; c, bovine serum albumin, 69; d, ovalbumin, 46; e, carbonic anhydrase, 29.

$\mathrm{SP}$ and $\mathrm{SP}^{\prime}$ are large and small subunits, respectively. 
positive periodate-Schiff staining; b) TLC analyses of dansyl hydrazone derivatives of sugars liberated on $0.3,0.6$, or $1 \mathrm{~N}$ TFA/ $100^{\circ} \mathrm{C} / 4 \mathrm{hr}$ treatment yielded three spots, and two of them were tentatively identified as galactose and arabinose dansyl hydrazones; c) analyses of hexose (cysteine-sulfuric acid) and pentose (ferric-orcinol) gave $1.3 \%$ and $1.6 \%$, respectively. Ovalbumin and BSA were used as the positive and negative controls, respectively, in these analyses. We therefore suggest that the larger and disconcerting values obtained by the gel filtration method are due to the differences in the glycosyl parts of enzyme molecules obtained from different cultivars. Whether these differences have effects on resistance
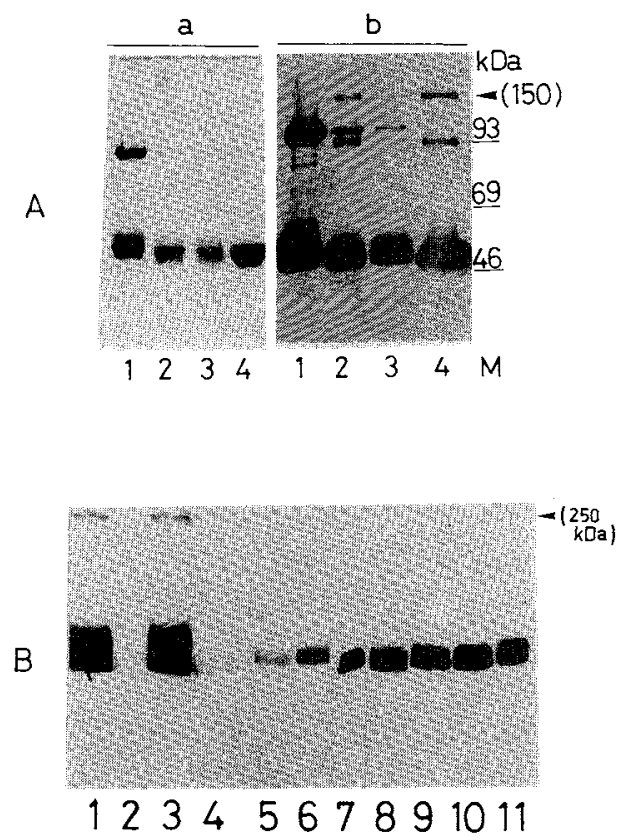

Fig. 4. Formation of a $250-\mathrm{kDa}$ SP and Changes of Subunit Patterns after a Long Period of Storage in $50 \%$ Glycerol at $-20^{\circ} \mathrm{C}$.

A. $7.5 \%$ SDS-PAGE: 1 , freshly purified T65 SP; $2, \mathrm{~T} 65$ SP stored for 10 months; 3 , freshly purified T57 SP; 4, T57 SP stored for 8 months. a, Coomassie blue stain; $b$, immunoblotting with $\mathrm{mAb} J 3 \mathrm{~b}$. $\mathrm{M}$, molecular mass markers, $\mathrm{kDa}$. The arrow indicates the $150-\mathrm{kDa}$ subunit. B. $10 \%$ disc-PAGE: Activity stain was done according to ref. 3. 1 and 3, stained in the presence of $0.3 \%$ soluble starch, for 30 and $60 \mathrm{~min}$, respectively. 2,4-11, stained without added starch for $0.5,1,2,4,7,10,15,24$, and $28 \mathrm{hr}$, respectively. against in vivo proteolysis merits future studies. The values found by the PAGE method were comparable to those reported so far for other dimeric forms of plant SP. ${ }^{1,3)}$ The calibration curve for molecular mass of the intact subunit and that of the main initial degradation product is presented in Fig. 3C.

When enzymes purified from roots of the two cultivars were stored in $50 \%$ glycerol at $-20^{\circ} \mathrm{C}$ for 8 to 10 months, the formation of a small amount of a high molecular mass form (250-kDa) of active enzyme that had an unusually large subunit, besides the $50-\mathrm{kDa}$ ones, of $150-\mathrm{kDa}$ could be observed. It can be considered as a product of transpeptidation between two intact SP subunits catalyzed by the contaminating protease that has a specific site of action at around the central part of the SP peptide. The formation of the unusually large subunit was significant in both $\mathrm{T} 65$ and

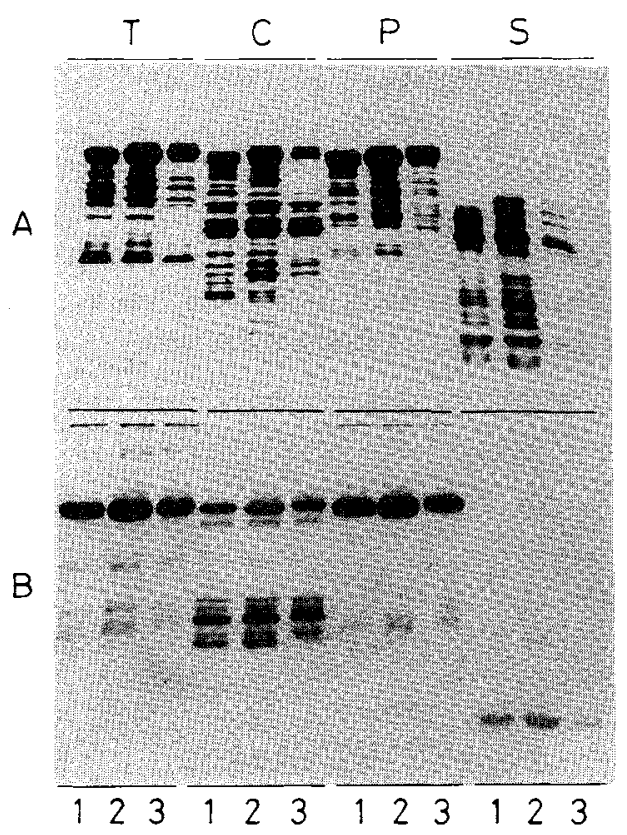

Fig. 5. Maps of Limited Proteolysis Fragments of T57 and T65 Phosphorylases Purified from Roots Freshly Harvested and Stored for 5 weeks.

15-20\% SDS-PAGE was used. 1 and 3, respectively from $\mathrm{T} 57$ and $\mathrm{T} 65$ roots stored at room temperature for 5 weeks; 2 , from freshly harvested $\mathrm{T} 57$ roots; $\mathrm{T}, \mathrm{C}, \mathrm{P}$, and $\mathrm{S}$ denote trypsin, chymotrypsin, protease $V_{8}$, and subtilisin, respectively. A, silver stain; $B$, immunoblotting with $\mathrm{mAb}$ G2c. 
T57 preparations, indicating that the protease was not deficient in $\mathrm{T} 65$, thus strengthening the view that the subunits of T65 SP were heterologous and the one that survived proteolysis after an extended period of storage was due to an inherent property different from the others. The more complex patterns of the immunoblot obtained for both rather fresh and extensively stored T65 SP preparations (Fig. 4A) may also support this view. The transpeptidation product retained the primed but not the unprimed enzyme activity (Fig. 4B). It is also noteworthy that the unprimed SP activity was confined to the lower molecular mass part of the enzyme cluster. Whether there is an enzyme domain the loss of it (as the result of degradation to a certain smaller molecular size) results in the loss of primed activity is an interesting problem to look into in the future.

When the limited proteolytic fragments of T65 and T57 enzymes were analyzed by the SDS-PAGE-Western blotting, similar but not completely identical patterns were obtained (Fig. 5). This indicates that the enzymes from two sources are highly homologous but have certain structural differences. We may thus conclude that the SP peptides, at least a part of them, of the two kinds of cultivar are coded by analogous but different genes.

\section{References}

1) T. Fukui, "Plant Phosphorylase: Structure and Function," ed. by T. Akazawa, T. Asahi, and H. Imaseki, The New Frontiers in Plant Biochemistry, Japan Scientific Societies Press, Tokyo, 1983, pp. $71-82$.

2) E. M. Schneider, J.-U. Becker and D. Volkmann, Planta, 151, 124 (1981).

3) T. C. Chang, S. C. Lee and J. C. Su, Agric. Biol. Chem., 51, 187 (1987).

4) S. M. Pan, T. C. Chang, R. H. Juang and J. C. Su, Plant Physiol., 88, 1154 (1988).

5) T. C. Chang, H. K. Wu, M. H. Chen and J. C. Su, Abstracts of Papers, The Second Joint Annual Conference of Biomedical Science, Taipei, March, 1987, p. 106.

6) E. M. Schneider, J.-U. Becker and D. Volkmann, Planta, 151, 124 (1981).

7) M. Ariki and T. Fukui, Biochim. Biophys. Acta, 386, 301 (1975).

8) R. H. Juang and J. C. Su, J. Chinese Biochem. Soc., 17, 42 (1988).

9) O. H. Lowry, N. J. Rosebrough, A. L. Farr and R. J. Randall, J. Biol. Chem., 193, 265 (1951).

10) M. S. Chern, Y. C. Mo, R. H. Juang and J. C. Su, J. Chinese Biochem. Soc., 19, 55 (1990).

11) P. Lambin, Anal. Biochem., 85, 114 (1978).

12) H. Y. Chan, T. Y. Ling, R. H. Juang, I. N. Ting, H. Y. Sung and J. C. Su, Plant Physiol, 94, 1456 (1990).

14) S. J. Gerbrandy, V. Shankar, K. N. Shivaram and H. Stegemann, Phytochemistry, 14, 2331 (1975).

15) R. H. Richardson and N. K. Matheson, Phytochemistry, 16, 1875 (1977). 